\title{
Urachal carcinoma with liver, lung, and brain metastases: benefit of a new combination chemotherapy (bevacizumab, 5-fluorouracil, irinotecan) - case report
}

This article was published in the following Dove Press journal:

Clinical Oncology in Adolescents and Young Adults

28 May 2011

Number of times this article has been viewed

\section{J Dekoninck' \\ $P$ Demetter ${ }^{2}$ \\ Filip Geurs' \\ R De Loecker ${ }^{3}$ \\ 'Department of Medical Oncology, Regional Hospital Sint Maria, Halle, Belgium; '2Department of Pathology, Hopital Erasme - Université Libre de Bruxelles (ULB), Anderlecht, Belgium; ${ }^{3}$ Department of Laboratory, Regional Hospital Sint Maria, Halle, Belgium}

Correspondence: Filip Geurs Regional Hospital Sint Maria, Ziekenhuislaan 100, BI500 Halle, Belgium Tel +32 23636610

Fax +3223636264

Email g@regzhsintmaria.be

\begin{abstract}
After failure of cisplatin-based chemotherapy for metastatic urachal carcinoma, a 16-year-old patient presents with diffuse liver-, lung-, and brain metastases. The K-ras mutation is present in the liver as well as in the primary resected specimen. The regimen was therefore changed to bevacizumab, 5-fluorouracil, and irinotecan. This led to a partial response which lasted 7 months.
\end{abstract}

Keywords: K-ras mutation, urachal cancer, adenocarcinoma

\section{Introduction}

It is estimated that urachal carcinoma constitutes less than $1 \%$ of all bladder tumors ${ }^{1-3}$ and approximately $10 \%$ of all primary adenocarcinomas of the bladder. ${ }^{4}$ In contrast with the much more frequent transitional cell cancers of the bladder, urachal cancer typically occurs in a younger patient population, with many reports indicating a median age at diagnosis of approximately $47-56$ years. ${ }^{2-4}$ While some investigators report a predominantly male patient population, ${ }^{5}$ others suggest a more balanced distribution between male and female patients. ${ }^{3,4}$ Unfortunately, there are many patients who present with metastatic disease which currently is not likely to be curable. There is no standard chemotherapy regimen for these patients. This case report builds on prior experiences in chemotherapy to report a new way of treating a patient with refractory metastatic disease.

\section{Clinical case}

The then 16-year-old male patient was diagnosed with T4 urachal bladder carcinoma, with presentation of hematuria. Initially (January 2009), he underwent a total cystectomy for this T4 neoplasm. The pathology report documented urachal adenocarcinoma. Six months later, salvage surgery with retroperitoneal dissection was performed for recurrent metastasis of the iliacal lymph nodes. Nine months after initial surgery, liver metastases were documented, and subsequently three courses of paclitaxel, ifosfamide, and cisplatin were administered. This led to a partial response of the liver metastases after 9 weeks. These treatments were done at a university hospital nearby. Due to asthenia and fatigue, the patient and his parents decided to stop this type of chemotherapy. Fourteen months after diagnosis, the then 17-year-old patient was admitted with seizures due to massive cerebral metastases, responding to valproic acid. Subsequent staging showed massive liver metastases as well as large mediastinal lymph nodes (Figure 1). 


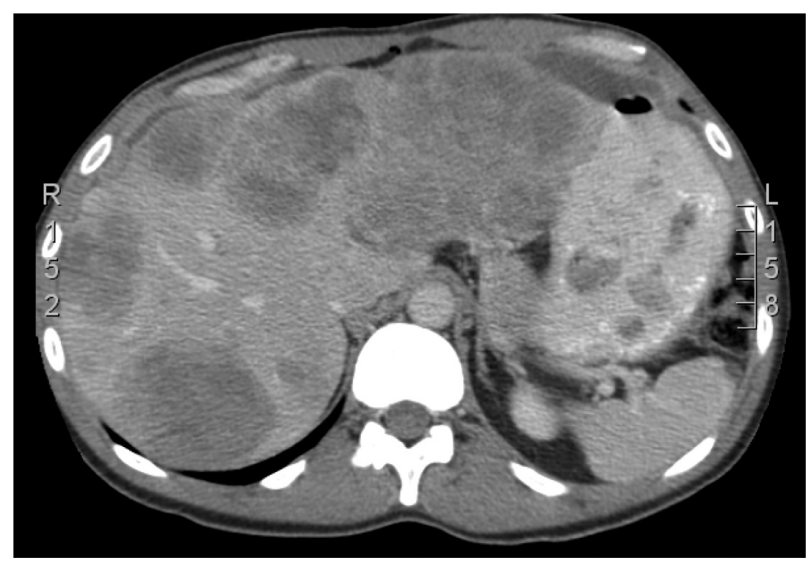

Figure I Liver metastases - before treatment.

A liver biopsy confirmed the specific histology of urachal carcinoma; a sample was sent for K-ras mutation, and the results showed that the mutation was present.

After cerebral radiotherapy $(10 \times 3 \mathrm{~Gy})$, a combination of bevacizumab, 5-fluorouracil, and irinotecan was administered. The regimen consisted of l-leukovorin $250 \mathrm{mg} / \mathrm{m}^{2}$, irinotecan $50 \mathrm{mg} / \mathrm{m}^{2}$, followed by 5 - fluorouracil $2 \mathrm{~g} / \mathrm{m}^{2} / 24 \mathrm{~h}$, six weeks out of eight. ${ }^{6-8}$ Bevacizumab was given at the dose of $5 \mathrm{mg} / \mathrm{kg}$ every 2 weeks. His liver pain decreased after 1 week. After an initial surge in tumor markers (carcinoembryonic antigen [CEA] and neuron-specific enolase [NSE]), a normalization was observed after 4 weeks (Figure 2). This is the first case reporting the use of NSE monitoring in metastatic urachal cancer; CEA as tumor marker was already known. Computed tomography (CT) scan of liver and lung metastases confirmed a cystic transformation, corresponding with a partial response (Figures 3 and 4). After 5.5 months of treatment, ascites developed; analysis showed neoplastic cells, and subsequent staging showed pulmonary and hepatic progression as well as neoplastic ascites (Figure 5). Palliative care at home was set up and the patient died at home 7 months after starting second-line chemotherapy.

\section{Discussion}

\section{Characteristics of urachal carcinoma}

The urachus (median umbilical ligament) extends from bladder dome to the umbilicus. It develops during embryogenesis from a diverticulum within the hindgut (allantois). As the cloaca divides and develops into the bladder anteriorly and the rectum posteriorly, the allantois is obliterated to form the urachus. ${ }^{9}$ The urachus is a three layered structure consisting of an outer muscular layer, a middle connective tissue layer, and an inner layer lined with transitional epithelium. ${ }^{10}$ In $30 \%$ of adults, congenital urachal anomalies, such as fistulae, cysts, sinuses and diverticulae, are present. The presence of these remnants increase the risk of urachal cancer. ${ }^{11}$

Urachal cancer commonly presents with hematuria and mucinuria. ${ }^{12}$ Given its extraperitoneal location, diagnosis is generally late. Common metastatic sites include lung, pelvic lymph nodes, and bone. ${ }^{5}$ Diagnosis of urachal cancer is best made by cystoscopy, although urinary cytology is positive in $38 \%$ of cases. ${ }^{5}$ Ultrasound and CT are useful in assessing the extent of local and distant disease.

During diagnosis, it is important to differentiate between urachal and nonurachal bladder adenocarcinoma, as staging, prognosis, and treatment differ. ${ }^{4}$

Tumor markers including CEA and CA 19-9 have been elevated in multiple case reports. In one series, 13 of 22

Tumor markers evolution

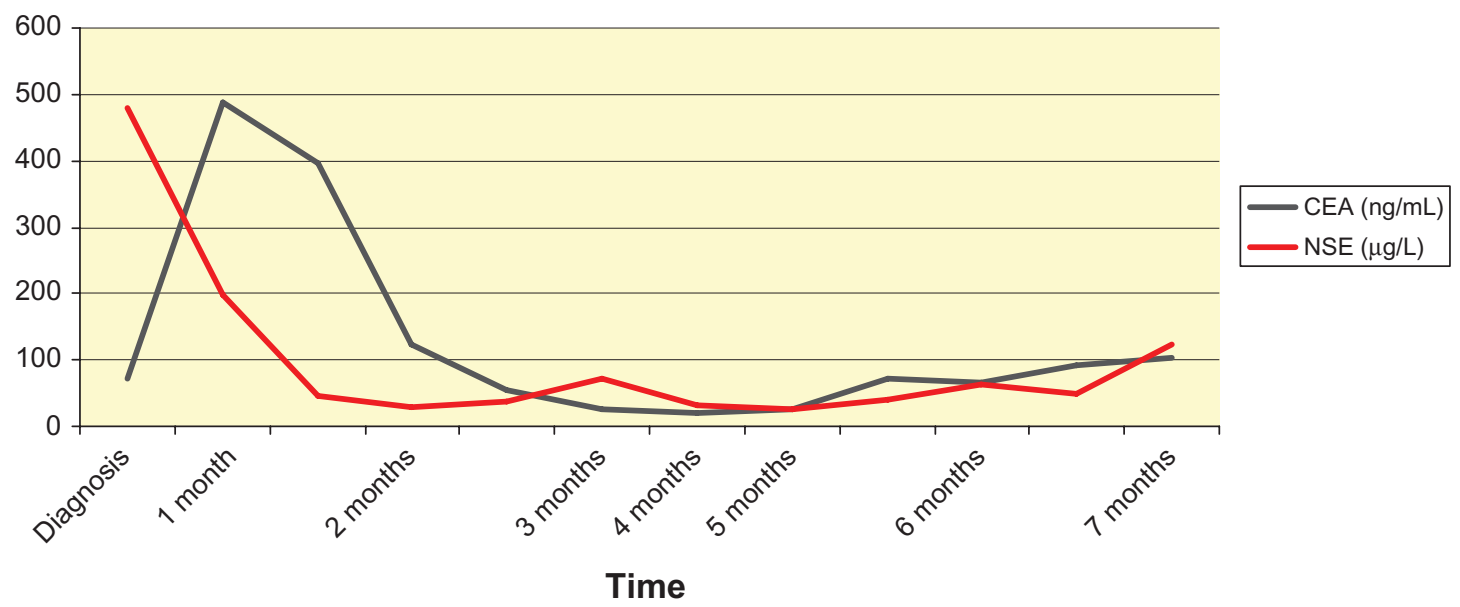

Figure 2 Evolution of tumor markers CEA and NSE during treatment

Abbreviations: CEA, carcinoembryonic antigen; NSE, neuron-specific enolase. 


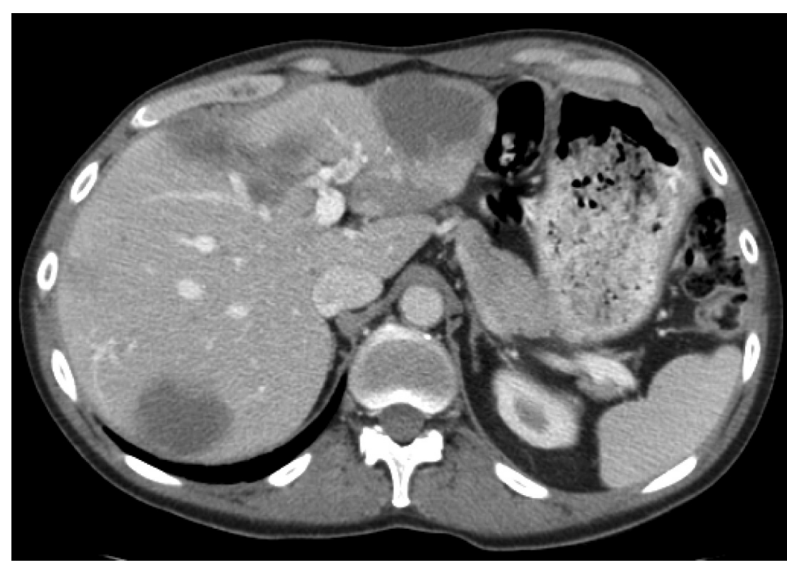

Figure 3 Liver 3 months after treatment.

patients demonstrated an elevated CEA, and five of these patients had a CEA decrease parallel with an objective response to chemotherapy. ${ }^{3}$ Our patients demonstrated a significant decrease of NSE, which was the first marker to drop as an indication of the effectiveness of the chemotherapy regimen. Staging systems used for traditional bladder cancers cannot be applied to urachal cancer, as the pattern of growth is intrinsically different. ${ }^{5}$

Historically, radical cystectomy was the operation of choice for resectable urachal cancer. However, due to umbilical involvement in $7 \%$ of patients, urachal cancer surgery now consists of a partial cystectomy with en bloc resection of the umbilicus and urachal ligament. Compared with radical cystectomy, this has significantly reduced the risk of local relapse and improved quality of life..$^{5,13}$

\section{Chemotherapy options}

With no current chemotherapy standard for the treatment of urachal carcinoma, most reports of chemotherapy response have been anecdotal at best. Previous reports describe the

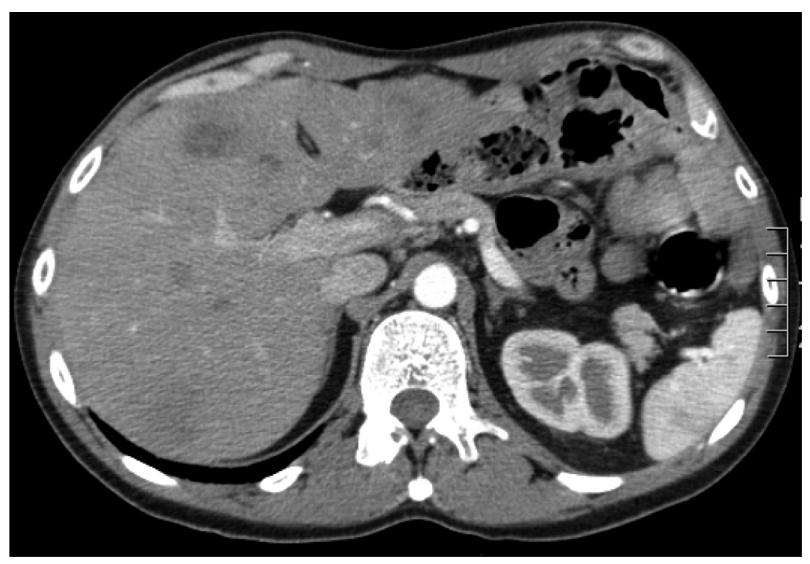

Figure 4 Liver 4 months after treatment. treatment of urachal tumors as 'invariably unsatisfactory' with a prognosis that was 'uniformly' poor. ${ }^{14}$ The first patient series appeared in 1985 when Logothetis and colleagues reported two partial responses out of three patients treated with 5-fluorouracil, doxorubicin, and mitomycin. ${ }^{15}$ Unfortunately, none of these responses were durable. A first MDACC report ${ }^{3}$ gave a 33\% response rate, using newer chemotherapy regimens which were cisplatin based. There was one additional responder using a combination of paclitaxel, methotrexate, and cisplatin (TMP), and no response in five patients treated with methotrexate, vinblastine, adriamycin, and cisplatin (M-VAC) ${ }^{16,17}$ Other reports suggest a single case of long-term survival with gemcitabin and cisplatin (GC), ${ }^{5} \mathrm{~S}-1$, cisplatin, ${ }^{18}$ and cisplatin with irinotecan. ${ }^{19}$ The most aggressive cisplatin based combination came from the Memorial Sloan-Kettering Cancer Center (NY, USA). These authors reported results on the first clinical trial in nontransitional cell histology including small cell, squamous, adenocarcinomas and urachal tumor. With their combination of ifosfamide, paclitaxel, and cisplatin (ITP), there was no response out of six patients treated for urachal carcinoma. ${ }^{20}$

The case reports from the 21 st century are based on the efficacy of irinotecan or oxaliplatin based chemotherapy regimens which are analogous to the treatment of colon cancer. Urachal adenocarcinomas are often histologically similar to adenocarcinomas from the gastrointestinal tract such as the colon or the stomach. ${ }^{21}$

All effective drugs and drug combinations used in metastatic colon cancer were used and proven effective in more recent cases of urachal carcinoma: oxaliplatin and irinotecan, in combination with 5- fluorouracil.

The use and efficacy of oxaliplatin-based chemotherapy has been recently reported in a case report. Partial response was documented in a 60-year-old patient with diffuse metastatic disease. ${ }^{22}$

Irinotecan is a topoisomerase I inhibitor that disrupts cell division by interfering with DNA replication. Irinotecan has demonstrated preclinical activity in adenocarcinomas from a variety of tumor types including gastric, colorectal, pancreatic, lung, and breast carcinomas. ${ }^{23}$ Currently, irinotecan in combination with 5-fluorouracil/leucovorin with or without bevacizumab is indicated as first-line therapy for metastatic colorectal cancer. ${ }^{24}$ Irinotecan has also demonstrated efficacy for metastatic gastric cancer in combination with 5-fluorouracil/leucovorin or cisplatin. ${ }^{25,26}$

The clear activity of IFL, an American version of irinotecan based colon cancer chemotherapy regimen, ${ }^{24}$ was 


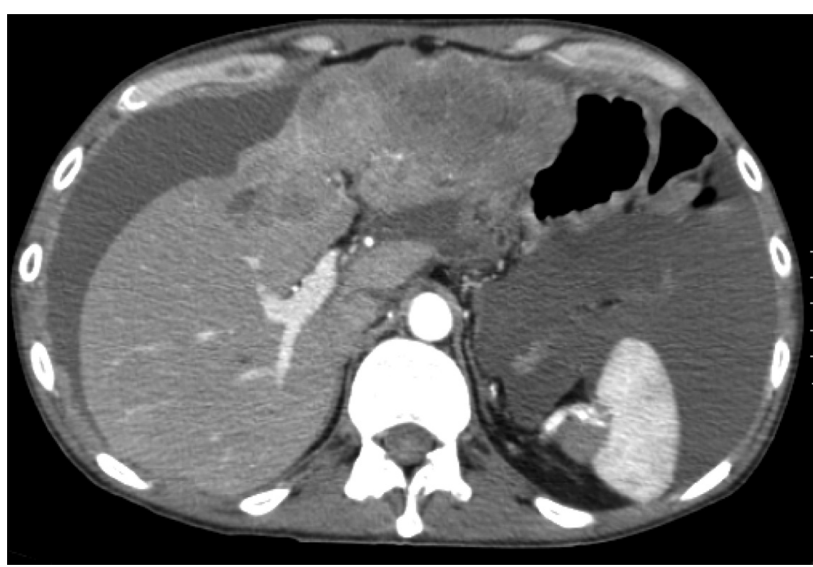

Figure 5 Liver almost 7 months after treatment.

recently illustrated in an MDAC case report. The patient had two successive complete responses in metastatic recurrences of disseminated urachal cancer, even in disease which was refractory to radiotherapy. ${ }^{21}$ Other case reports showed similar efficacy. ${ }^{27,28}$

The most recent development in metastatic colorectal cancer was the association of the targeted treatment (antiepidermal growth factor receptor [EGFR]-antibodies) with the irinotecan +5 -fluorouracil chemotherapy regimen. ${ }^{29}$

Over the last 2 years, it has become clear that the efficacy of the targeted treatment is limited to patients with the absence of EGFR-mutation in their initial colon cancer. These tumors are therefore called 'wild type'. This has led to an important change in international guidelines. ${ }^{29}$

Angiogenesis inhibiting monoclonal antibodies remain active in K-ras mutant subtypes of metastatic colorectal cancer. ${ }^{30}$ This knowledge was applied in our case, where K-ras mutation was documented in the liver metastases (and also in the primary tumor specimen). We therefore chose to add bevacizumab to the 5 -fluorouracil + irinotecan combination, although literature on its use in metastatic urachal cancer was lacking.

This new combination of the bevacizumab-irinotecan5 -fluorouracil regimen was shown to be effective in second-line treatment of metastatic urachal cancer leading to partial response of diffuse metastases. This partial response of metastatic disease was clinically evident shortly after the start of treatment and was proven effective, even after failure of an aggressive first-line chemotherapy regimen. Toxicity was acceptable, and overall survival of 7 months in second-line is analogous to survival times reported in other case reports, with less important cancer burden, and where chemotherapy was only reported in first-line therapy.

\section{Conclusion}

We report the first case of metastatic urachal cancer responding to bevacizumab/5-fluorouracil/irinotecan combination chemotherapy in a 17-year-old patient. A partial response was obtained after 9 weeks, and overall survival was 7 months, unprecedented in second line.

\section{Disclosure}

The authors report no conflicts of interest in this work.

\section{References}

1. Sheldon CA, Clayman RV, Gonzalez R, et al. Malignant urachal lesions. J Urol. 1984;131:1-8.

2. Henly DR, Farrow GM, Zincke H. Urachal cancer: role of conservative surgery. Urology. 1993;42:635-639.

3. Siefker-Radtke AO, Gee J, Shen Y, et al. Multimodality management of urachal carcinoma. The MDAnderson Cancer Center experience. J Urol. 2003;169:1295-1298.

4. Whright JL, Porter MP, Li CI, et al. Differences in survival among patients with urachal and nonurachal adenocarcinomas of the bladder. Cancer. 2006;107:721-728.

5. Ashley RA, Inman BA, Sebo TJ, et al. Urachal carcinoma: clinicopathologic features and long-term outcomes of an aggressive malignancy. Cancer. 2006;107:712-720.

6. Hofheinz R, Hartung G, Samel S, et al. Adding weekly irinotecan to high-dose 5-fluorouracil and folinic acid after failure for firstline HD-5-FU/FA in advanced colorectal cancer - a phase II study. Anticancer Drugs. 2002;13:999-1004.

7. Douillard J, Cunningham D, Roth AD. Irinotecan combined with fluorouracil compared with fluorouracil alone as first-line treatment for metastatic colorectal cancer: a multicentre randomised controlled trial. Lancet. 2000;355:1041-1047.

8. Verwimp J, Geurs F, Ponette S, et al. Complete response of a metastatic gastroesophageal adenocarcinoma on irinotecan-based chemotherapy in a dialysis patient. Int J Nephrol Renovasc Dis. 2010;3:61-64.

9. Ashley RA, Inman BA, Routh JC, et al. Urachal anomalies: a longitudinal study of urachal remnants in children and adults. J Urol. 2007;178: 1615-1618.

10. Siefker-Radtke A. Urachal carcinoma: surgical and chemotherapy options. Expert Rev Anticancer Ther. 2008;6:1715-1721.

11. Yu JS, Kim KW, Lee HJ, et al. Urachal remnant diseases: spectrum of CT and US findings. Radiographics. 2001;21(2):451-461.

12. Molina JR, Quevedo JF, Furth AF, et al. Predictors of survival from urachal cancer. Cancer. 2007;110(11):2434-2440.

13. Herr HW, Bochner BH, Sharp D, et al. Urachal carcinoma: contemporary surgical outcomes. J Urol. 2007;178(1):74-78.

14. Lane V. Prognosis in carcinoma of the urachus. Eur Urol. 1976;2: 282-283.

15. Logothetis CJ, Samuels ML, Ogden S. Chemotherapy for adenocarcinomas of bladder and urachal origin: 5-fluorouracil, doxorubin, and mitomycin-C. Urology. 1985;26:252-255.

16. Siefker-Radtke AO, Czerniak BA, Dinney CP, Millikan RE. Uncommon cancers of the bladder. In: Raghavan D, Brecher ML, Johnson DH, Meropol NJ, Moots PL, Rose PG, editors. Textbook of Uncommon Cancer. Hoboken, NJ: John Wiley \& Sons; 2006:18-26.

17. Siefker-Radtke AO, Millikan RE, Tu SM, et al. Phase III trial of fluorouracil, interferon $\alpha-2 b$, and cisplatin versus methotrexate, vinblastine, doxorubicin, and cisplatin in metastatic and unresectable urothelial cancer. J Clin Oncol. 2002;20:1361-1367.

18. Kojima Y, Yamada Y, Kamisawa H, et al. Complete response of a recurrent advanced urachal carcinoma treated by $\mathrm{S}-1 /$ cisplatin combination chemotherapy. Int J Urol. 2006;13:1123-1125. 
19. Kume H, Tomita $\mathrm{K}$, Takahashi $\mathrm{S}$, et al. Irinotecan as a new agent for urachal cancer. Urol Int. 2006;76:281-282.

20. Galsky MD, Iasanos A, Mironov S, et al. Prospective trial of ifosfamide, paclitaxel, and cisplatin (ITP) in patients with advanced non-transitional cell carcinomas of the urothelial tract. J Clin Oncol. 24(18):4542.

21. Mohile S, Schleider L, Petrylak D. Treatment of metastatic urachal carcinoma in an elderly woman. Nature Oncol. 2008;5:55-58.

22. Tran B, McKendrick J. Metastatic urachal cancer responding to FOLFOX chemotherapy. Can J Urol. 2010;2:5120-5123.

23. Rothenberg ML, Kuhn JH, Burris HA, et al. Phase I and pharmacokinetic trial of weekly CPT-11. J Clin Oncol. 1993;11:2194-2204.

24. Hurwitz HI, Fehrenbacher L, Hainsworth JD, et al. Bevacizumab in combination with flurouracil and leucovorin: an active regimen for First-line metastatic colorectal cancer. J Clin Oncol. 2005;23: 3502-3508.

25. Pozzo C, Barone C, Szanto J, et al. Irinotecan in combination with 5 -fluorouracil and folinic acid or with cisplatin in patients with advanced gastric or oesophageal-gastric junction adenocarcinoma: results of a randomized phase II study. Ann Oncol. 2004;15:1773-1781.
26. Bouche O, Raoul JL, Bonnetain F, et al. Randomized multicenter phase II trial of a biweekly regimen of fluorouracil and leucovorin (LV5FU2), LV5FU2 plus cisplatin, or LV5FU2 plus irinotecan in patients with previously untreated metastatic gastric cancer: a Federation Francophone de Cancerologie Digestive Group Study - FFCD 9803. J Clin Oncol. 2004;22:4319-4328.

27. Tazi E, Lalya I, Tazi MF, et al. Treatment of metastatic urachal adenocarcinoma in a young woman: a case report. Cases J. 2009;2:9145.

28. Kikuchi M, Kamei S, Morirama Y, et al. Case of urachal cancer treated by neoadjuvant chemotherapy with FOLFOX 4 (oxaliplatin, 5-FU and leukovolin). Hinyokika Kiyo. 2008;54(8):557-559.

29. Allegra CJ, Jessup JM, Somerfield MR, et al. American Society of Clinical Oncology provisional clinical opinion: testing for KRAS gene mutations in patients with metastatic colorectal carcinoma to predict response to anti-epidermal growth factor receptor monoclonal antibody therapy. J Clin Oncol. 2009;27:2091-2096.

30. Grothey A, Galanis E. Targeting angiogenesis: progress with antiVEGF- treatment with large molecules. Nat Rev Clin Oncol. 2009;6(9): $507-518$.

\section{Publish your work in this journal}

Clinical Oncology in Adolescents and Young Adults is an international, peer-reviewed, open access journal publishing original research, reports, editorials, reviews and commentaries on all aspects of epidemiology, diagnosis and treatment of cancers in adolescents and young adults.
Visit http://www.dovepress.com/testimonials.php to read real quotes from published authors. 